\title{
Pathologic Response Rates for Breast Cancer Stages as a Predictor of Outcomes in Patients Receiving Neoadjuvant Chemotherapy Followed by Breast-Conserving Surgery
}

Chang-Yun Lu

Taipei Medical University

Ho-Min Chen

Asia University

Szu-Yuan Wu ( $\nabla$ szuyuanwu5399@gmail.com )

Taipei Medical University https://orcid.org/0000-0001-5637-558X

\section{Research}

Keywords: breast cancer, neoadjuvant chemotherapy, breast-conserving surgery, pathologic response rates, survival

Posted Date: November 20th, 2020

DOl: https://doi.org/10.21203/rs.3.rs-111293/v1

License: (c) (1) This work is licensed under a Creative Commons Attribution 4.0 International License.

Read Full License 


\section{Abstract}

PURPOSE: To determine easy-to-use predictors of overall survival (OS), locoregional recurrence (LRR), and distant metastasis (DM) in patients with breast invasive ductal carcinoma (IDC) receiving neoadjuvant chemotherapy (NACT) followed by breast-conserving surgery (BCS), we obtained pathologic response rates (PRRs) for combined primary and nodal diseases (American Joint Committee on Cancer [AJCC] stages) from clinical and pathologic reports, and we used these as predictors.

PATIENTS AND METHODS: We enrolled patients with IDC who had received NACT followed by BCS. Cox regression analysis was used to calculate hazard ratios (HRs) and confidence intervals (Cls) for the patients' PRRs; other independent predictors were controlled for or stratified in the analysis.

RESULTS: We analyzed 1047 patients with IDC (611, 260, and 176 patients in clinical stages IIB, IIIA, and IIIB-C, respectively) receiving NACT and BCS. After multivariate Cox regression analyses, the adjusted HRs (aHRs; $95 \% \mathrm{Cl}$ ) in patients with pathologic complete response (ypTON0) were $0.26(0.13-0.56), 0.36$ (0.15-0.85), and 0.15 (0.08-0.31) for all-cause mortality, LRR, and DM, respectively. The aHRs $(95 \% \mathrm{Cl})$ in patients with downstaging of AJCC stages were 0.55 (0.35-0.89), 0.91 (0.62-0.96), and 0.63 (0.43$0.91)$ for all-cause mortality, LRR, and DM, respectively. The aHRs $(95 \% \mathrm{Cl})$ in patients with upstaging of AJCC stages were 1.77 (1.06-2.24), 1.08 (1.03-1.82), and 1.19 (1.07-2.01) for all-cause mortality, LRR, and DM, respectively.

CONCLUSION: The impacts of AJCC-stage PRRs are useful predictive tools and strong predictors for OS, LRR, and DM in patients with breast IDC receiving NACT followed by BCS.

\section{Introduction}

Neoadjuvant chemotherapy (NACT) is associated with high rates of clinical response and a greater likelihood that cosmetically acceptable surgery can be performed.[1, 2] For example, patients who are not candidates for breast-conserving surgery (BCS) may become eligible after NACT; those with limited nodepositive diseases (CN1) may be appropriate candidates for more limited axillary surgery after NACT.[2, 3] However, NACT alone has not been shown to improve disease-free survival (DFS) or overall survival (OS) compared with the same treatment administered after surgery.[1]

Among patients treated with NACT, the achievement of a pathologic complete response (pCR) to surgery is prognostically significant.[4-7] Pathologic assessment of the breast and axillary nodes is performed to determine the presence and extent of residual invasive disease after NACT completion. The achievement of pCR in the breast and axilla (ypT0 \& ypN0) correlates with improved survival.[6, 8] Through the pooled analysis of the Collaborative Trials in Neoadjuvant Breast Cancer with mature follow-up data, we aimed to characterize the relationship between $\mathrm{pCR}$ and long-term outcomes.[9] Achieving a pCR in both the breast and the axilla was associated with improved event-free survival (EFS) and OS compared with a pCR (ypT0 \& ypN0) in the breast alone (ypT0).[9] However, studies have associated a pCR with superior OS in patients with breast cancer receiving NACT followed by either total mastectomy (TM) or BCS,[4-7, 
9-12] but sufficient data do not support the association between PCR and survival benefits in patients receiving NACT followed by BCS. In addition, pathologic response after NACT includes not only pCR but also partial response, disease stability, and disease progression.[10] No study has shown the predictive ability of pathologic response in patients with breast cancer receiving NACT followed with mastectomy, particularly with respect to BCS.

In the past, the residual cancer burden (RCB) score has provided a means for assessing the extent of residual invasive disease in the tumor bed and the extent of residual nodal involvement after NACT.[10] However, RCB is not widely used as a predictor for survival in current clinical practice because no consensus for reading RCB exists among pathologists. Thus, we used the clinical and pathological American Joint Committee on Cancer (AJCC) tumor, node, and metastasis system after NACT to obtain simple pathologic response rates (PRRs) as predictors for patients with breast cancer receiving NACT followed by BCS. For patients with breast cancer receiving NACT, according to their PRRs and the applicability of poor prognostic factors, BCS might be used as a more aggressive adjuvant, or consolidative chemotherapy might subsequently be necessary.

\section{Patients And Methods}

In this study, we established a cohort of patients with breast invasive ductal carcinoma (IDC) by using data from the Taiwan Cancer Registry database (TCRD). We enrolled patients with breast IDC diagnoses between January 1, 2007, and December 31, 2015. The follow-up duration was from the index date to December 31, 2016. The Cancer Registry database of the Collaboration Center of Health Information Application contains detailed cancer-related information of patients, including clinical stages, treatment modalities, pathologic data, irradiation doses, hormone receptor (HoR) status, human epidermal growth factor receptor 2 (HER2) status, and chemotherapy regimens used.[13-21] In the study, we included women with breast IDC receiving NACT followed by BCS. Our protocols were reviewed and approved by the Institutional Review Board of Taipei Medical University. The diagnoses of the enrolled patients were confirmed through their pathologic data, and patients who received a new diagnosis of breast IDC were confirmed to have no other types of cancer. Patients with a diagnosis of breast IDC who had received NACT followed by BCS, who were aged $\geq 20$ years, and whose AJCC clinical cancer stages were III to IIIC were included. Moreover, initial AJCC clinical staging and pathologic staging were recorded in the TCRD. The breast cancer stages were assessed according to the AJCC, seventh edition. Patients with metastasis, missing sex data, age $<20$ years, receipt of nonstandard adjuvant whole breast radiotherapy (where standard RT means RT to treat the entire breast with 1.8- to 2-Gy daily fractions for 4.5 to 5 weeks, signifying a total dose of 45 to $50 \mathrm{~Gy}$ ), unclear differentiation of tumor grade, unclear pathologic response, missing HoR status, missing HER2 status, or unclear staging were excluded. Furthermore, we excluded patients with unclear NACT regimens, receipt of fewer than four NACT cycles, ill-defined nodal surgery, and missing hospital types[22] (academic center or community hospitals) in our cohort. PRRs were classified into upstages (clinical stage upgrade to advanced pathologic stage), equal stages (clinical stage equal to pathologic stage), downstages (clinical stage down to relatively early pathologic stages), and pCRs (absence of residual invasive cancer). HoR positivity was defined as $\geq 1 \%$ of tumor 
cells demonstrating positive nuclear staining through immunohistochemistry,[23] and HER2 positive was defined as an immunohistochemistry score $3+$ or fluorescence in situ hybridization ratio $\geq 2 .[22,24]$ Finally, we enrolled patients with breast IDC receiving NACT followed by BCS and categorized them into clinical AJCC stage groups. The index date was the date of breast cancer diagnosis. Comorbidities were scored using the Charlson comorbidity index (CCI). $[25,26]$ Only comorbidities observed 6 months before the index date were considered; comorbidities that were included were identified according to the main International Classification of Diseases, Ninth Revision, Clinical Modification (ICD-9-CM) diagnosis codes for the first admission or the main diagnosis codes associated with more than two visits to the outpatient department.

After adjustment for confounders, the Cox proportional method was used to model the time from the index date to all-cause mortality, LRR, and DM among patients who had received NACT followed by BCS. In the multivariate analysis, hazard ratios (HRs) were adjusted for the AJCC stage PRRs, initial clinical stage, age, diagnosis year, CCI score, differentiation, NACT regimen, nodal surgery, adjuvant RT, HoR status, HER2 status, and academic hospital status. Stratified analyses for the various initial clinical stages were performed to evaluate the predictors of all-cause death; furthermore, in the multivariate analysis, we used the PRRs of AJCC stage, initial clinical stage, age, diagnosis year, CCl score, differentiation, NACT regimen, nodal surgery, adjuvant RT, HoR status, HER2 status, and academic hospital. Multivariate analysis with stratification according to the PRRs for $\mathrm{T}$ stages or $\mathrm{N}$ stages was conducted to evaluate the impact on all-cause death, LRR, DM, and DFS of NACT followed by BCS. All analyses were performed using SAS (version 9.3; SAS, Cary, NC, USA). A two-tailed value of $P<0.05$ was considered statistically significant.

\section{Results}

The final cohort eligible for further analysis consisted of 1047 patients $(611,260$, and 176 patients in clinical stages IIB, IIIA, and IIIB-IIIC, respectively). The patient characteristics are summarized in Table 1. Initial clinical stages were found to exert no statistically significant effects in terms of $\mathrm{CCl}$ score, tumor differentiation, diagnosis year, use of adjuvant RT, HoR status, HER2 receptor status, or academic hospital status (Table 1). The disease was in the advanced stages IIIB to IIIC for more of a greater number of elderly patients in the cohort. More patients receiving NACT were diagnosed between 2011 and 2015 than between 2007 and 2010. More patients with breast IDC in the advanced stages IIIA to IIIC received taxanebased NACT regimens and axillary lymph node dissection (ALND). In addition, patients with breast cancer in the more advanced clinical stages IIIA to IIIC exhibited more AJCC-stage, T-stage, and N-stage pCRs or downstaging. Risks for all-cause death, LRR, and DM were significantly higher in advanced clinical stages, such as stage IIIA to IIIC, compared with in stage IIB (Table 1).

Table 1. Characteristics of Patients Receiving Neoadjuvant Chemotherapy Followed by Breast-Conserving Surgery in Various AJCC Clinical Stages 


\begin{tabular}{|c|c|c|c|c|c|}
\hline Variable & & $\begin{array}{c}\text { Clinical } \\
\text { Stage IIB } \\
(N=611)\end{array}$ & $\begin{array}{c}\text { Clinical } \\
\text { Stage IIIA } \\
(N=260)\end{array}$ & $\begin{array}{c}\text { Clinical Stage } \\
\text { IIIB-IIIC } \\
(N=176)\end{array}$ & $P$ value \\
\hline \multirow[t]{4}{*}{ Age } & Mean (SD) & $46.4(9.8)$ & $46.0(9.5)$ & $50.9(10.2)$ & $<0.0001$ \\
\hline & $\begin{array}{l}\text { Median (Q1, } \\
\text { Q3) }\end{array}$ & $46(39,54)$ & $46(39,53)$ & $51(43,59)$ & \\
\hline & $20-49$ & 389 (63.7\%) & $163(62.7 \%)$ & 78 (44.3\%) & $<0.0001$ \\
\hline & $50+$ & 222 (36.3\%) & 97 (37.3\%) & 98 (55.7\%) & \\
\hline \multirow[t]{2}{*}{ Diagnosis year } & $2007-2010$ & 94 (15.4\%) & 66 (25.4\%) & 34 (19.3\%) & 0.1231 \\
\hline & 2011-2015 & 517 (84.6\%) & $194(74.6 \%)$ & $142(80.7 \%)$ & \\
\hline \multirow[t]{3}{*}{ CCI score } & 0 & 521 (85.3\%) & 217 (83.5\%) & $146(83.0 \%)$ & 0.3921 \\
\hline & 1 & $67(11.0 \%)$ & 37 (14.2\%) & 21 (11.9\%) & \\
\hline & $2+$ & $23(3.8 \%)$ & $6(2.3 \%)$ & $9(5.1 \%)$ & \\
\hline \multirow[t]{3}{*}{ Differentiation } & I & $30(5.0 \%)$ & $14(5.4 \%)$ & $10(5.7 \%)$ & 0.1575 \\
\hline & II & 329 (53.8\%) & $127(48.8 \%)$ & 96 (54.5\%) & \\
\hline & III & $252(41.2 \%)$ & 119 (45.8\%) & 70 (39.8\%) & \\
\hline \multirow[t]{4}{*}{ NACT regimen } & Taxanes & 271 (44.4\%) & $113(43.5 \%)$ & 86 (48.9\%) & 0.0068 \\
\hline & Anthracycline & 199 (32.6\%) & 68 (26.2\%) & 39 (22.2\%) & \\
\hline & Both & 97 (15.9\%) & 66 (25.4\%) & $39(22.2 \%)$ & \\
\hline & Neither & $44(7.2 \%)$ & $13(5.0 \%)$ & $12(6.8 \%)$ & \\
\hline \multirow[t]{2}{*}{ Nodal surgery } & ALND & 491 (80.4\%) & 225 (86.5\%) & $152(86.4 \%)$ & 0.0285 \\
\hline & SLNB & $120(19.6 \%)$ & 35 (13.5\%) & 24 (13.6\%) & \\
\hline Adjuvant RT & & $564(92.3 \%)$ & 243 (93.5\%) & $160(90.9 \%)$ & 0.6143 \\
\hline $\begin{array}{l}\text { Hormone receptors } \\
\text { positivity }\end{array}$ & & $330(54.0 \%)$ & 135 (51.9\%) & $82(46.6 \%)$ & 0.2200 \\
\hline $\begin{array}{l}\text { HER2 receptor } \\
\text { positivity }\end{array}$ & & $202(33.1 \%)$ & $106(40.8 \%)$ & 57 (32.4\%) & 0.0691 \\
\hline \multirow[t]{2}{*}{ Academic hospital } & Yes & 407 (66.6\%) & $165(63.5 \%)$ & $100(56.8 \%)$ & 0.0556 \\
\hline & No & $204(33.4 \%)$ & 95 (36.5\%) & $76(43.2 \%)$ & \\
\hline \multirow{4}{*}{$\begin{array}{l}\text { AJCC stage rate } \\
\text { response }\end{array}$} & $\mathrm{pCR}$ & $101(16.5 \%)$ & 55 (21.2\%) & $24(13.6 \%)$ & $<0.0001$ \\
\hline & Downstages & 349 (57.1\%) & 155 (59.6\%) & $122(69.3 \%)$ & \\
\hline & Equal stages & 74 (12.1\%) & 38 (14.6\%) & $26(14.8 \%)$ & \\
\hline & Upstages & $87(14.2 \%)$ & $12(4.6 \%)$ & $4(2.3 \%)$ & \\
\hline
\end{tabular}




\begin{tabular}{|c|c|c|c|c|c|}
\hline \multicolumn{2}{|l|}{ Variable } & $\begin{array}{c}\text { Clinical } \\
\text { Stage IIB } \\
(N=611)\end{array}$ & $\begin{array}{c}\text { Clinical } \\
\text { Stage IIIA } \\
(N=260)\end{array}$ & $\begin{array}{c}\text { Clinical Stage } \\
\text { IIIB-IIIC } \\
(N=176)\end{array}$ & $P$ value \\
\hline \multirow{4}{*}{$\begin{array}{l}\text { T-stage rate } \\
\text { response }\end{array}$} & T-pCR & $114(18.7 \%)$ & $64(24.6 \%)$ & $25(14.2 \%)$ & $<0.0001$ \\
\hline & Downstages & $336(55.0 \%)$ & $164(63.1 \%)$ & $118(67.0 \%)$ & \\
\hline & Equal stages & 155 (25.4\%) & 32 (12.3\%) & 33 (18.8\%) & \\
\hline & Upstages & $6(1.0 \%)$ & $0(0.0 \%)$ & $0(0.0 \%)$ & \\
\hline \multirow{5}{*}{$\begin{array}{l}\mathrm{N} \text {-stage rate } \\
\text { response }\end{array}$} & N-pCR & 305 (49.9\%) & 135 (51.9\%) & 70 (39.8\%) & $<0.0001$ \\
\hline & & & 34 (13.1\%) & 42 (23.9\%) & \\
\hline & Downstages & $0(0.0 \%)$ & & & \\
\hline & Equal stages & $214(35.0 \%)$ & $65(25.0 \%)$ & $46(26.1 \%)$ & \\
\hline & Upstages & $92(15.1 \%)$ & $26(10.0 \%)$ & $18(10.2 \%)$ & \\
\hline \multicolumn{2}{|c|}{ Mean follow-up time, months (SD) } & $64.7(26.0)$ & $59.7(28.0)$ & $51.0(26.2)$ & \\
\hline \multicolumn{2}{|l|}{ All-cause death } & $54(8.8 \%)$ & $44(16.9 \%)$ & $32(18.2 \%)$ & $<0.0001$ \\
\hline \multicolumn{2}{|l|}{$\begin{array}{l}\text { Local regional } \\
\text { recurrence }\end{array}$} & $60(9.8 \%)$ & $27(10.4 \%)$ & $26(14.8 \%)$ & $<0.0001$ \\
\hline \multicolumn{2}{|c|}{ Distant metastasis } & $95(15.5 \%)$ & $63(24.2 \%)$ & $41(23.3 \%)$ & $<0.0001$ \\
\hline
\end{tabular}

RT, radiation therapy; T, tumor; N, nodal; NACT, neoadjuvant chemotherapy; BCS, breast-conserving surgery; HR, hormone receptor; HER2, human epidermal growth factor receptor 2; pCR, pathologic complete response; ALND, axillary lymph node dissection; SNLB, sentinel lymph node biopsy; SD, standard deviation; CCI, Charlson comorbidity index; AJCC, American Joint Committee on Cancer; IQR, interquartile range; T-pCR, pathologic complete response in the breast; N-pCR, pathologic complete response in regional lymph nodes

According to the multivariate Cox regression analysis, the AJCC stage PRRs were significantly independent predictors of all-cause death, LRR, and DM (Tables 2). Both pCR and downstaging for AJCC stages were strong predictors for decreased risk of all-cause death, LRR, and DM. Upstaging of AJCC stages was a strong predictor for increased risk of all-cause death, LRR, and DM. AJCC stage PCRs (ypTON0) were the strongest predictor for decreased risk of all-cause death, LRR, and DM. The adjusted HRs (aHRs; 95\% confidence interval [CI]) of pCR were 0.26 (0.13-0.56), $0.36(0.15-0.85)$, and 0.15 (0.08$0.31)$ for all-cause mortality, LRR, and DM, respectively. The aHRs $(95 \% \mathrm{Cl})$ for AJCC downstaging were 0.55 (0.35-0.89), 0.91 (0.62-0.96), and 0.63 (0.43-0.91) for all-cause mortality, LRR, and DM, respectively. The aHRs $(95 \% \mathrm{Cl})$ for upstaging of AJCC stages were 1.77 (1.06-2.24), 1.08 (1.03-1.82), and 1.19 (1.07-2.01) for all-cause mortality, LRR, and DM, respectively (Table 2). Multivariate Cox regression analyses indicated that the upstaging of AJCC stages, no use of adjuvant RT, initial AJCC clinical stages of IIIA to IIIC, and HoR negativity to be poor prognostic factors for OS (Table 2). The AJCC stage pCR or downstaging, adjuvant RT, and HoR positivity were independently good prognostic factors for OS. In addition, according to multivariate analysis, no use of adjuvant RT treatment, AJCC clinical stages IIIA to IIIC, post-NACT upstaging of AJCC stages, and HER2 positivity were poor prognostic factors 
for LRR (Table 2). Moreover, multivariate analysis revealed that AJCC clinical stages IIIA to IIIC, postNACT upstaging of AJCC stages, no use of adjuvant RT treatment, HoR negativity, and HER2 positivity were independently poor prognostic factors for DM after multivariate analysis. Table 3 presents the independent predictors of all-cause death for patients receiving NACT followed by BCS stratified by initial clinical stages after multivariate analysis. The aHRs $(95 \% \mathrm{Cl})$ of the pCRs for AJCC stages were 0.24 (0.06-0.94), $0.19(0.06-0.66)$, and $0.51(0.11-0.93)$ for all-cause mortality among initial clinical stages IIB, IIIA, and IIIB to IIIC, respectively. The aHRs $(95 \% \mathrm{Cl})$ for the downstaging of AJCC stages were 0.57 (0.26-0.98), $0.40(0.19-0.84)$, and $0.76(0.24-0.99)$ for all-cause mortality among the initial clinical stages IIB, IIIA, and IIIB to IIIC, respectively. Moreover, the aHRs $(95 \% \mathrm{Cl})$ for the upstaging of AJCC stages were 2.04 (1.18-4.76), 1.07 (1.01-4.41), and 2.42 (1.25-13.21) for all-cause mortality among the initial clinical stages IIB, IIIA, and IIIB to IIIC, respectively. Adjuvant RT and HoR negativity were independent risk factors of all-cause death for stages IIB to IIIC. HER2 positivity was an independent poor prognostic factor of all-cause death among clinical stages IIIA to IIIC (Table 3).

Table 2. Multivariable Analysis for All Causes of Death, Locoregional Recurrence, and Distant Metastasis for Patients Receiving Neoadjuvant Chemotherapy Followed by BreastConserving Surgery 


\begin{tabular}{|c|c|c|c|c|c|c|c|}
\hline & \multicolumn{2}{|c|}{ All-cause Death } & \multicolumn{2}{|c|}{ LRR } & \multicolumn{2}{|l|}{$\overline{\mathrm{DM}}$} \\
\hline & & $\begin{array}{cc}\text { HR } & (95 \% \\
& \text { CI })\end{array}$ & $P$ value & $\begin{array}{cc}\mathrm{HR} \quad(95 \% \\
\mathrm{CI})\end{array}$ & $P$ value & $\begin{array}{c}\text { HR }(95 \% \\
\text { CI) }\end{array}$ & $P$ value \\
\hline \multirow{4}{*}{$\begin{array}{l}\text { AJCC stage } \\
\text { rate response }\end{array}$} & Equal stages & ref & $<0.0001$ & ref & 0.0736 & ref & $<0.0001$ \\
\hline & $\mathrm{pCR}$ & $\begin{array}{c}0.26(0.13- \\
0.56)\end{array}$ & & $\begin{array}{c}0.36(0.15- \\
0.85)\end{array}$ & & $\begin{array}{c}0.15(0.08- \\
0.31)\end{array}$ & \\
\hline & Downstages & $\begin{array}{c}0.55(0.35- \\
0.89)\end{array}$ & & $\begin{array}{r}0.91(0.62- \\
0.96)\end{array}$ & & $\begin{array}{r}0.63(0.43- \\
0.91)\end{array}$ & \\
\hline & Upstages & $\begin{array}{r}1.77(1.06- \\
2.24)\end{array}$ & & $\begin{array}{r}1.08 \text { (1.03- } \\
1.82)\end{array}$ & & $\begin{array}{r}1.19 \text { (1.07- } \\
2.01)\end{array}$ & \\
\hline \multirow[t]{4}{*}{ Clinical stage } & IIB & ref & $<0.0001$ & ref & $<0.0001$ & ref & $<0.0001$ \\
\hline & IIIA & $\begin{array}{c}2.20(1.43- \\
3.37)\end{array}$ & & $\begin{array}{c}1.32(1.13- \\
1.75)\end{array}$ & & $\begin{array}{c}2.06(1.46- \\
2.91)\end{array}$ & \\
\hline & IIIB & $\begin{array}{c}2.31(1.31- \\
4.05)\end{array}$ & & $\begin{array}{c}1.38(1.14- \\
1.96)\end{array}$ & & $\begin{array}{r}1.72 \text { (1.07- } \\
2.75)\end{array}$ & \\
\hline & IIIC & $\begin{array}{r}3.65(1.92- \\
6.94)\end{array}$ & & $\begin{array}{r}1.56(0.78- \\
3.15)\end{array}$ & & $\begin{array}{r}2.57 \text { (1.48- } \\
4.48)\end{array}$ & \\
\hline \multirow[t]{2}{*}{ Age } & $20-49$ & ref & 0.7605 & ref & 0.5630 & ref & 0.3455 \\
\hline & $50+$ & $\begin{array}{c}0.94(0.64- \\
1.39)\end{array}$ & & $\begin{array}{c}1.14(0.73- \\
1.77)\end{array}$ & & $\begin{array}{c}0.85(0.62- \\
1.18)\end{array}$ & \\
\hline \multirow[t]{2}{*}{ Diagnosis year } & 2007-2010 & ref & 0.3178 & ref & 0.9117 & ref & 0.6856 \\
\hline & 2011-2015 & $\begin{array}{c}0.81(0.53- \\
1.23)\end{array}$ & & $\begin{array}{c}0.93(0.60- \\
1.78)\end{array}$ & & $\begin{array}{c}0.93(0.65- \\
1.32)\end{array}$ & \\
\hline \multirow[t]{3}{*}{ CCI score } & 0 & ref & 0.2232 & ref & 0.8237 & ref & 0.1102 \\
\hline & 1 & $\begin{array}{r}1.05(0.91- \\
2.30)\end{array}$ & & $\begin{array}{r}1.09 \text { (0.48- } \\
1.67)\end{array}$ & & $\begin{array}{r}1.12(1.02- \\
2.26)\end{array}$ & \\
\hline & $2+$ & $\begin{array}{r}1.14(0.26- \\
1.89)\end{array}$ & & $\begin{array}{r}1.26(0.48- \\
3.30)\end{array}$ & & $\begin{array}{r}1.33 \text { (0.63- } \\
2.85)\end{array}$ & \\
\hline \multirow[t]{3}{*}{ Differentiation } & III & ref & 0.4668 & ref & 0.5892 & ref & 0.3709 \\
\hline & II & $\begin{array}{r}0.93 \text { (0.67- } \\
1.58)\end{array}$ & & $\begin{array}{c}1.03(0.64- \\
1.65)\end{array}$ & & $\begin{array}{c}1.01(0.71- \\
1.44)\end{array}$ & \\
\hline & I & $\begin{array}{r}0.46(0.16- \\
1.35)\end{array}$ & & $\begin{array}{c}0.26(0.03- \\
1.91)\end{array}$ & & $\begin{array}{c}0.77(0.34- \\
1.72)\end{array}$ & \\
\hline \multirow[t]{3}{*}{ NACT regimen } & Anthracycline & ref & 0.1944 & ref & 0.6845 & ref & 0.0701 \\
\hline & Taxanes & $\begin{array}{r}0.69(0.44- \\
1.06)\end{array}$ & & $\begin{array}{c}0.92 \text { (0.57- } \\
1.47)\end{array}$ & & $\begin{array}{c}0.64(0.46- \\
1.01)\end{array}$ & \\
\hline & Both & $\begin{array}{r}0.68(0.41- \\
1.12)\end{array}$ & & $\begin{array}{c}0.68(0.36- \\
1.28)\end{array}$ & & $\begin{array}{c}0.40(0.25- \\
1.03)\end{array}$ & \\
\hline
\end{tabular}


Neither

$0.53(0.25-$

1.13)

Nodal surgery ALND

SLNB

Adjuvant RT

Hormone

receptors

positivity

HER2 receptor

positivity

Academic Yes

hospital ref

$1.13(0.63-$

2.04) ref 1.18)
$0.96(0.44-$

2.12)
$1.24(0.76-$ 2.01)
(0.22- < 0.0001
0.42
(0.23- 0.0033
$0.75)$

$$
0.57)
$$
$0.09(0.06-<0.0001$
$0.12)$

0.3591 ref

0.3719

$1.19(0.81-$

$0.71(0.44-$

2.38)

1.16)
$0.57(0.39-0.0033$
$0.86(0.56-0.4933$
1.32)
0.83 )
$0.85(0.63-0.0133$
0.97)

$1.08(0.52-0.2420$

1.62

(1.06- 0.0245

1.54

2.09)

2.46)

0.0054

0.3698 ref

0.2560 ref

0.4967

No

$0.84(0.56-$

$0.78(0.50-$

$0.90(0.66-$

1.24)

1.20)

1.23)

HR, hazard ratio; CI, confidence interval; RT, radiation therapy; NACT, neoadjuvant chemotherapy; HER2, human epidermal growth factor receptor 2; ALND, axillary lymph node dissection; SLNB, sentinel lymph node biopsy; CCI, Charlson comorbidity index; AJCC, American Joint Committee on Cancer; LRR, locoregional recurrence; DM, distant metastasis; $\mathrm{pCR}$, pathologic complete response; ref, reference group

Table 3. Multivariable Analysis for All Causes of Death for Patients Receiving Neoadjuvant Chemotherapy Followed by Breast-Conserving Surgery, Stratified by Clinical Stage 


\begin{tabular}{|c|c|c|c|c|c|c|c|}
\hline & \multicolumn{2}{|c|}{ Stage IIB } & \multicolumn{2}{|c|}{ Stage IIIA } & \multicolumn{2}{|c|}{ Stage IIIB-IIIC } \\
\hline & & $\begin{array}{cc}\text { HR } & (95 \% \\
& \text { CI })\end{array}$ & $P$ value & $\begin{array}{cc}\text { HR } & (95 \% \\
& \text { CI })\end{array}$ & $P$ value & $\begin{array}{cc}\text { HR } & (95 \% \\
& \text { CI })\end{array}$ & $P$ value \\
\hline \multirow{4}{*}{$\begin{array}{l}\text { AJCC stage rate } \\
\text { response }\end{array}$} & Equal stages & ref & 0.0004 & ref & 0.0265 & ref & 0.0185 \\
\hline & $\mathrm{pCR}$ & $\begin{array}{c}0.24(0.06- \\
0.94)\end{array}$ & & $\begin{array}{c}0.19(0.06- \\
0.66)\end{array}$ & & $\begin{array}{c}0.51(0.11- \\
0.93)\end{array}$ & \\
\hline & Downstages & $\begin{array}{c}0.57(0.26- \\
0.98)\end{array}$ & & $\begin{array}{c}0.40(0.19- \\
0.84)\end{array}$ & & $\begin{array}{c}0.76(0.24- \\
0.99)\end{array}$ & \\
\hline & Upstages & $\begin{array}{c}2.04(1.18- \\
4.76)\end{array}$ & & $\begin{array}{c}1.07(1.01- \\
4.41)\end{array}$ & & $\begin{array}{r}2.42(1.25- \\
13.21)\end{array}$ & \\
\hline \multirow[t]{2}{*}{ Age } & $20-49$ & ref & 0.4547 & ref & 0.2286 & ref & 0.7392 \\
\hline & $50+$ & $\begin{array}{r}1.26(0.69- \\
2.31)\end{array}$ & & $\begin{array}{r}0.63(0.30- \\
1.34)\end{array}$ & & $\begin{array}{c}1.15(0.50- \\
2.64)\end{array}$ & \\
\hline \multirow[t]{2}{*}{ Diagnosis year } & 2007-2010 & ref & 0.1481 & ref & 0.5048 & ref & 0.0772 \\
\hline & 2011-2015 & $\begin{array}{r}0.62(0.32- \\
1.19)\end{array}$ & & $\begin{array}{c}1.30(0.60- \\
2.83)\end{array}$ & & $\begin{array}{c}0.47(0.20- \\
1.09)\end{array}$ & \\
\hline \multirow[t]{3}{*}{ CCI score } & 0 & ref & 0.3333 & ref & 0.4243 & ref & 0.9980 \\
\hline & 1 & $\begin{array}{c}1.66(0.76- \\
3.63)\end{array}$ & & $\begin{array}{r}1.65(0.78- \\
3.52)\end{array}$ & & $\begin{array}{c}1.04(0.35- \\
3.09)\end{array}$ & \\
\hline & $2+$ & $\begin{array}{r}1.74(0.56- \\
5.41)\end{array}$ & & $\begin{array}{r}1.76(0.77- \\
4.52)\end{array}$ & & $\begin{array}{r}1.50(0.47- \\
3.12)\end{array}$ & \\
\hline \multirow[t]{3}{*}{ Differentiation } & III & ref & 0.9587 & ref & 0.4594 & ref & 0.3469 \\
\hline & II & $\begin{array}{r}0.91 \text { (0.47- } \\
1.75)\end{array}$ & & $\begin{array}{r}1.28(0.55- \\
2.96)\end{array}$ & & $\begin{array}{c}0.44(0.16- \\
1.17)\end{array}$ & \\
\hline & I & $\begin{array}{c}0.69(0.15- \\
3.12)\end{array}$ & & $\begin{array}{c}0.55(0.11- \\
2.87)\end{array}$ & & $\begin{array}{c}0.39(0.10- \\
1.97)\end{array}$ & \\
\hline \multirow[t]{4}{*}{ NACT regimen } & Anthracycline & ref & 0.6900 & ref & 0.3307 & ref & 0.8830 \\
\hline & Taxanes & $\begin{array}{r}0.87(0.45- \\
1.67)\end{array}$ & & $\begin{array}{r}0.53(0.23- \\
1.22)\end{array}$ & & $\begin{array}{r}0.93(0.33- \\
2.59)\end{array}$ & \\
\hline & Both & $\begin{array}{r}0.74(0.32- \\
1.71)\end{array}$ & & $\begin{array}{c}0.50(0.20- \\
1.23)\end{array}$ & & $\begin{array}{c}1.14(0.36- \\
3.62)\end{array}$ & \\
\hline & Neither & $\begin{array}{r}0.52(0.16- \\
1.61)\end{array}$ & & $\begin{array}{r}0.89(0.26- \\
3.05)\end{array}$ & & $\begin{array}{c}0.49(0.06- \\
4.26)\end{array}$ & \\
\hline \multirow[t]{2}{*}{ Nodal surgery } & ALND & ref & 0.9459 & ref & 0.5412 & ref & 0.3265 \\
\hline & SLNB & $\begin{array}{c}1.09(0.44- \\
2.74)\end{array}$ & & $\begin{array}{c}1.76(0.64- \\
4.79)\end{array}$ & & $\begin{array}{c}0.60(0.15- \\
2.31)\end{array}$ & \\
\hline
\end{tabular}

Adjuvant RT

$0.45(0.20-0.03410 .30(0.12-0.01080 .11(0.04-<0.0001$ 0.95)

0.76 )

0.29 ) 
Hormone

receptor

positivity

HER2 receptor

positivity

Academic

Yes

0.51

(0.251.01)

ref

hospital
$0.94)$

0.03000 .46

(0.22- 0.0326

0.94 )

$(0.31-0.0015$

0.89 )
$0.95(0.51-$
$0.95(0.51-$
$1.79)$
No
$0.48(0.21-$
$1.51(0.98-$
1.08)
5.85)

HR, hazard ratio; CI, confidence interval; RT, radiation therapy; NACT, neoadjuvant chemotherapy; HER2, human epidermal growth factor receptor 2; ALND, axillary lymph node dissection; SLNB, sentinel lymph node biopsy; CCI, Charlson comorbidity index; AJCC, American Joint Committee on Cancer; pCR, pathologic complete response; ref, reference group

Because the PRRs for AJCC stages were significant predictors for all-cause death, LRR, and DM, we also estimated the effect of PRRs for T stages and $N$ stages on all-cause death, LRR, DM, and DFS in patients with breast cancer receiving NACT followed by BCS. Supplemental Fig. 1 shows that T-stage PRRs were significant predictors for all-cause death, LRR, DM, and DFS in patients with breast IDC receiving NACT followed by BCS. Only the upstaging of T stages was not a significant predictor for LRR. T-stage pCRs or downstaging were good prognostic factors for all-cause death, LRR, DM, and DFS in patients with breast IDC receiving NACT followed by BCS. The upstaging of T stages after NACT followed by BCS was a significantly poor prognostic factor for all-cause death, DM, and DFS. The N-pCR was a significant predictor for all-cause death, LRR, DM, and DFS in patients receiving NACT followed by BCS.

Nevertheless, the downstaging of $\mathrm{N}$ stages was not a statistically significant predictor for all-cause death, LRR, DM, and DFS in patients with breast cancer receiving NACT followed by BCS (Supplemental Fig. 2). The upstaging of $\mathrm{N}$ stages was a significantly poor prognostic factor for all-cause death, DM, and DFS but was not a significant predictor for LRR.

\section{Discussion}

NACT is the systemic treatment of breast cancer prior to definitive surgical therapy such as TM or BCS with ALND or SLNB. $[2-7,9,10]$ Although all systemic therapy for nonmetastatic invasive breast cancer is intended to reduce the risk of $\mathrm{DM}$, the purpose of administering chemotherapy prior to surgery is to downstage tumors and provide information regarding treatment response.[3, 11, 27-29] Typically, neoadjuvant treatment is administered in the form of chemotherapy such as anthracycline-based chemotherapy, taxane-based chemotherapy, or both as basic regimens.[30-32] No data or research have indicated an association between pathologic response and survival outcomes such as all-cause death, LRR, and DM in patients with breast IDC receiving NACT followed by BCS. Most studies have shown that pCR in patients with breast cancer receiving NACT indicates superior survival outcomes compared with those non-pCR patients with breast cancer receiving NACT followed by either TM or BCS.[4-7, 9-12] 
However, no other pathologic responses, such as partial pathologic response or progressive change of pathologic response, function as predictors for survival outcomes in patients with breast cancer receiving NACT followed by BCS. Studies have shown associations between pCR and EFS or OS,[4-7, 9-12] but no further data are available on the associations of other pathologic responses, based on AJCC stages (downstages, upstages, or equal stages), with survival outcomes for OS, LRR, or DM. Therefore, the use of AJCC stage PRRs (equal stages, upstages, downstages, and PCR) as predictors in patients with breast IDC receiving NACT followed by BCS provides a valuable tool. The respective outcomes might indicate further adjuvant treatments. Our study is the first to demonstrate the real value of PRRs in patients with breast IDC receiving NACT followed by BCS. According to our findings (Table 2-3), in the future, physicians might consider subsequent alternative treatments such as further TM instead of BCS or adjuvant RT in patients with high LRR risk. In patients for whom PRRs indicate high DM risk, adjuvant or consolidative chemotherapy with innovative regimens might be considered for decreasing the risk of DM. Thus, the use of PRRs for patients with breast IDC receiving NACT followed by BCS, which acts as a simple predictive tool, is potentially valuable for identifying further treatment choices for patients with high LRR or DM risk. As such, aggressive treatments might be omitted in patients with low DM and LRR risk.

Multivariable analysis demonstrated the AJCC stage PRRs to be significant predictors for OS, LRR, and DM in patients with breast IDC receiving NACT followed by BCS (Table 2). Our study is the first study to show that AJCC stage PRRs are strong predictors in patients receiving NACT followed by BCS. In our study, we evaluated the predictive ability of PRRs not only for OS and EFS[4-7, 9-12] but also for LRR and DM in patients with breast IDC receiving NACT followed by BCS. Although studies have found the RCB to predict survival after NACT,[10,33] the complicated measurement system for the RCB, requiring consensus among particular pathologists, is not popularly used globally. Moreover, although the RCB is used as a predictor for DM or OS, it is not used for LRR, regardless of subsequent surgical procedures, such as TM, BCS, ALND, or SLNB, assessed in other research.[10, 33] In Table 2, the upstaging of AJCC stages after NACT is seen to be associated with high risk of all-cause death, LRR, and DM. Therefore, in the future, patients with breast IDC receiving NACT upstaging of AJCC stages might be considered for subsequent aggressive treatments such as TM, adjuvant RT, or innovative consolidative chemotherapy regimens.

Adjuvant RT is potentially valuable for patients with breast IDC receiving NACT followed by BCS (Tables 2-3). Adjuvant RT was shown to decrease the risk of all-cause death, LRR, and DM in these patients (Table 2). Physicians previously believed adjuvant RT to be beneficial for patients with breast $I D C$ receiving BCS on the basis of old studies in which patients with breast cancer received BCS without NACT[34]; however, no data had suggested that it exhibits real benefits for patients with breast IDC receiving NACT followed by BCS. The value of adjuvant RT after NACT followed by BCS has never been quantified, and clinical trials may be unable to resolve this. Our study showed that adjuvant RT is a significantly valuable treatment for the improvement of OS, LRR, or DM in patients with breast IDC receiving NACT followed by BCS, even in patients with AJCC stage pCR or downstaging (Table 2-3). This is the first study to show the survival benefits of adjuvant RT for patients with breast IDC receiving NACT 
followed by BCS. HoR positivity was also a significant predictor for OS, LRR, and DM in patients with breast IDC receiving NACT followed by BCS (Table 2). Our results, showing that HoR positivity is associated with superior survival outcomes in patients with breast cancer, corroborate those of other studies on various treatments. [35] Although HoR positivity was a good prognostic factor for OS, LRR, and DM in patients with breast cancer,[35] no data indicate HoR positivity to be a good prognostic factor in patients receiving NACT followed by BCS. Our study provides the best evidence that HoR positivity is a good prognostic factor for patients with breast IDC receiving NACT followed by BCS. In Table 2, HER2 positivity is seen to be a poor prognostic factor for LRR and DM in patients with breast IDC receiving NACT followed by BCS. Our findings are compatible with those of other studies that have similarly concluded that HER2 positivity is a risk factor for LRR and DM,[36],[37] although not specifically in patients with breast IDC receiving NACT followed by BCS. In our study, anthracycline- or taxane-based NACT regimens were not significant predictors of survival, as in the results of one meta-analysis that enrolled 1695 patients in nine trials.[38]

As seen in Table 2, multivariable analysis revealed initial clinical AJCC stages to be significant predictors of OS, LRR, and DM in patients with breast IDC receiving NACT followed by BCS. Therefore, clinical states are stratified in Table 3 to assess the value of PRRs as predictors in patients with breast IDC receiving NACT followed by BCS. As seen in Table 3, PRRs are strong predictors of OS, LRR, and DM in patients, even for stratified analysis of initial clinical AJCC stages. Multivariable analysis revealed that adjuvant RT, HoR positivity, and HER2 negativity were good prognostic factors of survival outcomes at all clinical stages (Table 3). Our findings indicate that PRRs are a simple and useful predictive tool, using clinical and pathologic AJCC stages from current pathologic practice for patients with breast IDC receiving NACT followed by BCS, regardless of their clinical stages. Adjuvant RT, HoR status, and HER2 status were also notable predictors for survival outcomes. For patients with breast IDC receiving NACT followed by BCS, adjuvant RT significantly decreased the risk of all-cause death, LRR, and DM, regardless of the initial clinical stage (Table 3).

As well as estimating the predictive ability of AJCC stage PRRs, we wished to estimate the predictive ability of T-stage PRRs (Supplemental Fig. 1) and N-stage PRRs (Supplemental Fig. 2). Multivariable analysis revealed that T-stage (ypT0) pCR and downstaging were good prognostic factors for OS, LRR, DM, and DFS but were nonsignificant for LRR. The findings show that T-stage PRRs were also good predictors for survival outcomes, although AJCC stage PRRs might be more useful for all OS, LRR, and DM outcomes than are T-stage PRRs (which are not significant predictors for LRR). In Supplemental Fig. 2, N-stage pCR (ypNO) is seen to be a significant predictor of OS, LRR, DM, and DFS. N-stage pCR indicated superior OS in patients with breast cancer receiving NACT, as in the results of other research. [39] The downstaging of $\mathrm{N}$ stages was not significant for OS, LRR, DM, or DFS, demonstrating that the downstaging of $\mathrm{N}$ stages was not a significant predictor of survival outcomes, in accord with other research results.[39] In addition, the upstaging of $\mathrm{N}$ stages was a poor prognostic factor for OS, DM, and DFS but was not a significant predictor of LRR. Taken together, the T-stage and N-stage PRRs both predicted partial survival outcomes in patients with breast IDC receiving NACT followed by BCS. 
Nevertheless, AJCC stage PRRs provided a more powerful predictive tool for all survival outcomes, including OS, LRR, and DM.

The strength of our research was that it constituted the first and largest cohort study in which the impacts of AJCC stage, T-stage, and N-stage PRRs on OS, LRR, and DM have been evaluated in patients with breast IDC receiving NACT followed by BCS. The NACT regimens used to treat patients were relatively homogenous in our study. No studies have used stratification of all predictors, including clinical stage, to evaluate in detail the impact of PRRs on treatment outcomes in patients with breast cancer receiving NACT followed by BCS. In our study, the poor prognostic factors of OS in breast cancer patients receiving NACT followed by BCS were no use of adjuvant RT, pre-NACT disease status in advanced clinical stages IIIA to IIIC, HoR negativity, and HER2 positivity (Table 2). Our simple tool, which used available AJCC clinical and pathologic staging records to obtain PRRs, differs from other research approaches wherein RCB was calculated as a continuous index combining pathologic measurements by trained pathologists. [33] Our PRRs were straightforward to use on the basis of staging records; were powerful for predicting OS, LRR, and DM in patients with breast IDC receiving NACT followed by BCS, regardless of clinical stages; and can be used to guide adjuvant treatment recommendations. In the future, the ability to identify the optimal NACT regimens for increasing pCRs or downstaging and for preventing the possibility of upstaging in patients with breast cancer receiving NACT will be valuable for precision medicine.[40, 41] For AJCC upstaging in patients with breast IDC receiving NACT, adjuvant chemotherapy or subsequent TM is greatly preferable to BCS because of the high risk of all-cause death, LRR, and DM (Table 2-3).

This study has some limitations. First, because all patients with breast IDC were enrolled from an Asian population, their ethnic susceptibility compared with non-Asian populations remains unclear. Hence, our results should be cautiously extrapolated to non-Asian populations. However, no evidence exists to demonstrate the differences between Asian and non-Asian populations in PRRs for patients with breast cancer receiving NACT followed by BCS. Second, the diagnoses for all comorbid conditions were based on ICD-9-CM codes. Nevertheless, the Taiwan Cancer Registry Administration randomly reviews charts and interviews patients to verify the accuracy of the diagnoses, and hospitals with outlier chargers or practices may be audited, and consequently, heavily penalized if malpractice or discrepancies are identified. Third, to prevent the formation of several subgroups, various regimens of NACT were not categorized separately during the analyses. Thus, the effects of specific neoadjuvant treatments remain unclear. Nevertheless, four cycles of NACT for each enrolled patient with breast IDC achieved consistent responses. Accordingly, to obtain crucial information on population specificity and disease occurrence, a large-scale randomized trial comparing carefully selected patients undergoing suitable treatments is essential. Finally, the TCRD contains no information regarding dietary habits, socioeconomic status, or body mass index, all of which may be risk factors for mortality. However, considering the magnitude and statistical significance of the effects observed in this study, these limitations are unlikely to have affected the conclusions.

\section{Conclusions}


PRRs provide an easy-to-use predictive tool, and they are strong predictors for OS, LRR, and DM in patients with breast IDC receiving NACT followed with BCS, regardless of the respective clinical stages. Adjuvant RT is necessary for patients with breast IDC receiving NACT followed by BCS.

\section{Abbreviations}

PRRs, pathologic response rates; RT, radiation therapy; T, tumor; N, nodal; OS, overall survival; LRR, locoregional recurrence; DM, distant metastasis; DFS, disease-free survival; EFS, event-free survival; NACT, neoadjuvant chemotherapy; TM, total mastectomy; BCS, breast-conserving surgery; HRs, hazard ratios; Cl, confidence interval; IDC, invasive ductal carcinoma; TCRD, Taiwan Cancer Registry database; AJCC, American Joint Committee on Cancer; HoR, hormone receptor; HER2, human epidermal growth factor receptor 2; CCI, Charlson comorbidity index; ICD-9-CM, International Classification of Diseases, Ninth Revision, Clinical Modification; pCR, pathological complete response; T stages, primary breast diseases; $\mathrm{N}$ stages, nodal diseases; ALND, axillary lymph node dissection; BCS, breast-conserving surgery; T-pCR, pathologic complete response in the breast; N-pCR, pathologic complete response in regional lymph nodes; RCB, residual cancer burden; ypT, postchemotherapy pathologic tumor stages; ypN, postchemotherapy pathologic nodal stages

\section{Declarations}

Ethics Approval and Consent: Our protocols were reviewed and approved by the Institutional Review Board of Taipei Medical University (TMU-JIRB No. 201712019).

Consent for Publication: Not applicable

Availability of Data and Material: The datasets supporting the study conclusions are included within this manuscript and its additional files.

\section{For Software:}

Project name: Not applicable

Project homepage: Not applicable

Archived version: Not applicable

Operating system(s): Not applicable

Programming language: Not applicable

Other requirements: Not applicable

License: Not applicable 
Any restrictions to use by non-scholars: Not applicable

Competing Interests: The authors have no potential conflicts of interest to declare. The datasets supporting the study conclusions are included in the manuscript.

Research Funding: Lo-Hsu Medical Foundation, LotungPoh-Ai Hospital, supports Szu-Yuan Wu's work (Funding Number: 10908 and 10909).

\section{Author Contributions}

Conception and Design: Chang-Yun Lu, MD; Ho-Min Chen, MS; Szu-Yuan Wu, MD, MPH, PhD

Financial Support: Lo-Hsu Medical Foundation, LotungPoh-Ai Hospital, supports Szu-Yuan Wu's work (Funding Number: 10908 and 10909).

Collection and Assembly of Data: Ho-Min Chen, MS; Szu-Yuan Wu, MD, MPH, PhD*

Data Analysis and Interpretation: Jiaqiang Zhang, MD, PhD; Ho-Min Chen, MS; Szu-Yuan Wu, MD, MPH, $\mathrm{PhD*}$

Administrative Support: Szu-Yuan Wu*

Manuscript Writing: Jiaqiang Zhang, MD, PhD; Chang-Yun Lu, MD; Ho-Min Chen, MS; Szu-Yuan Wu, MD, $\mathrm{MPH}, \mathrm{PhD}$

Final Approval of Manuscript: All authors

Acknowledgments: Lo-Hsu Medical Foundation, LotungPoh-Ai Hospital, supports Szu-Yuan Wu's work (Funding Number: 10908 and 10909).

Competing Interests: The authors have no potential conflicts of interest to declare. The datasets supporting the study conclusions are included within the manuscript.

Condensed Abstract: No study has comprehensively analyzed the impact of pathologic response rates (PRRs) for disease stages on overall survival (OS), locoregional recurrence (LRR), or distant metastasis (DM) in patients with breast invasive ductal carcinoma (IDC) receiving neoadjuvant chemotherapy (NACT) and breast-conserving surgery (BCS). This large cohort study was used to estimate the impact of changes in American Joint Committee on Cancer stages on OS, LRR, and DM in patients with IDC receiving NACT and BCS. Our data indicate that PRRs, determined using staging records, provide a straightforward means for predicting OS, LRR, and DM, regardless of the initial clinical stages of breast IDC. The results can be used to guide adjuvant treatment.

\section{References}


1. Early Breast Cancer Trialists' Collaborative G: Long-term outcomes for neoadjuvant versus adjuvant chemotherapy in early breast cancer: meta-analysis of individual patient data from ten randomised trials. Lancet Oncol 2018, 19:27-39.

2. Thompson AM, Moulder-Thompson SL: Neoadjuvant treatment of breast cancer. Ann Oncol 2012, 23 Suppl 10:x231-236.

3. Mamtani A, Barrio AV, King TA, Van Zee KJ, Plitas G, Pilewskie M, El-Tamer M, Gemignani ML, Heerdt AS, Sclafani LM, et al: How Often Does Neoadjuvant Chemotherapy Avoid Axillary Dissection in Patients With Histologically Confirmed Nodal Metastases? Results of a Prospective Study. Ann Surg Oncol 2016, 23:3467-3474.

4. Tanioka M, Shimizu C, Yonemori K, Yoshimura K, Tamura K, Kouno T, Ando M, Katsumata N, Tsuda $\mathrm{H}$, Kinoshita $\mathrm{T}$, Fujiwara $\mathrm{Y}$ : Predictors of recurrence in breast cancer patients with a pathologic complete response after neoadjuvant chemotherapy. Br J Cancer 2010, 103:297-302.

5. Kim MM, Allen P, Gonzalez-Angulo AM, Woodward WA, Meric-Bernstam F, Buzdar AU, Hunt KK, Kuerer HM, Litton JK, Hortobagyi GN, et al: Pathologic complete response to neoadjuvant chemotherapy with trastuzumab predicts for improved survival in women with HER2-overexpressing breast cancer. Ann Oncol 2013, 24:1999-2004.

6. Fayanju OM, Ren Y, Thomas SM, Greenup RA, Plichta JK, Rosenberger LH, Tamirisa N, Force J, Boughey JC, Hyslop T, Hwang ES: The Clinical Significance of Breast-only and Node-only Pathologic Complete Response (pCR) After Neoadjuvant Chemotherapy (NACT): A Review of 20,000 Breast Cancer Patients in the National Cancer Data Base (NCDB). Ann Surg 2018, 268:591-601.

7. Tan MC, Al Mushawah F, Gao F, Aft RL, Gillanders WE, Eberlein TJ, Margenthaler JA: Predictors of complete pathological response after neoadjuvant systemic therapy for breast cancer. Am J Surg 2009, 198:520-525.

8. Spring LM, Fell G, Arfe A, Sharma C, Greenup R, Reynolds KL, Smith BL, Alexander B, Moy B, Isakoff SJ, et al: Pathologic Complete Response after Neoadjuvant Chemotherapy and Impact on Breast Cancer Recurrence and Survival: A Comprehensive Meta-analysis. Clin Cancer Res 2020, 26:28382848.

9. Cortazar P, Zhang L, Untch M, Mehta K, Costantino JP, Wolmark N, Bonnefoi H, Cameron D, Gianni L, Valagussa $\mathrm{P}$, et al: Pathological complete response and long-term clinical benefit in breast cancer: the CTNeoBC pooled analysis. Lancet 2014, 384:164-172.

10. Symmans WF, Wei C, Gould R, Yu X, Zhang Y, Liu M, Walls A, Bousamra A, Ramineni M, Sinn B, et al: Long-Term Prognostic Risk After Neoadjuvant Chemotherapy Associated With Residual Cancer Burden and Breast Cancer Subtype. J Clin Oncol 2017, 35:1049-1060.

11. Schwartz GF, Hortobagyi GN: Proceedings of the consensus conference on neoadjuvant chemotherapy in carcinoma of the breast, April 26-28, 2003, Philadelphia, Pennsylvania. Cancer 2004, 100:2512-2532.

12. Cortazar P, Geyer CE, Jr.: Pathological complete response in neoadjuvant treatment of breast cancer. Ann Surg Oncol 2015, 22:1441-1446. 
13. Chang CL, Tsai HC, Lin WC, Chang JH, Hsu HL, Chow JM, Yuan KS, Wu ATH, Wu SY: Dose escalation intensity-modulated radiotherapy-based concurrent chemoradiotherapy is effective for advancedstage thoracic esophageal squamous cell carcinoma. Radiother Oncol 2017.

14. Chang WW, Hsiao PK, Qin L, Chang CL, Chow JM, Wu SY: Treatment outcomes for unresectable intrahepatic cholangiocarcinoma: Nationwide, population-based, cohort study based on propensity score matching with the Mahalanobis metric. Radiother Oncol 2018.

15. Chen TM, Lin KC, Yuan KS, Chang CL, Chow JM, Wu SY: Treatment of advanced nasopharyngeal cancer using low- or high-dose concurrent chemoradiotherapy with intensity-modulated radiotherapy: A propensity score-matched, nationwide, population-based cohort study. Radiother Oncol 2017.

16. Lin YK, Hsieh MC, Chang CL, Chow JM, Yuan KS, Wu ATH, Wu SY: Intensity-modulated radiotherapy with systemic chemotherapy improves survival in patients with nonmetastatic unresectable pancreatic adenocarcinoma: A propensity score-matched, nationwide, population-based cohort study. Radiother Oncol 2018.

17. Lin YK, Hsieh MC, Wang WW, Lin YC, Chang WW, Chang CL, Cheng YF, Wu SY: Outcomes of adjuvant treatments for resectable intrahepatic cholangiocarcinoma: Chemotherapy alone, sequential chemoradiotherapy, or concurrent chemoradiotherapy. Radiother Oncol 2018.

18. Yen YC, Hsu HL, Chang JH, Lin WC, Chang YC, Chang CL, Chow JM, Yuan KS, Wu ATH, Wu SY: Efficacy of thoracic radiotherapy in patients with stage IIIB-IV epidermal growth factor receptormutant lung adenocarcinomas who received and responded to tyrosine kinase inhibitor treatment. Radiother Oncol 2018.

19. Lin WC, Ding YF, Hsu HL, Chang JH, Yuan KS, Wu ATH, Chow JM, Chang CL, Chen SU, Wu SY: Value and application of trimodality therapy or definitive concurrent chemoradiotherapy in thoracic esophageal squamous cell carcinoma. Cancer 2017.

20. Yen YC, Chang JH, Lin WC, Chiou JF, Chang YC, Chang CL, Hsu HL, Chow JM, Yuan KS, Wu ATH, Wu SY: Effectiveness of esophagectomy in patients with thoracic esophageal squamous cell carcinoma receiving definitive radiotherapy or concurrent chemoradiotherapy through intensity-modulated radiation therapy techniques. Cancer 2017, 123:2043-2053.

21. Wu SY, Fang SC, Shih HJ, Wen YC, Shao YJ: Mortality associated with statins in men with advanced prostate cancer treated with androgen deprivation therapy. Eur J Cancer 2019, 112:109-117.

22. Bahreini F, Soltanian AR, Mehdipour P: A meta-analysis on concordance between immunohistochemistry (IHC) and fluorescence in situ hybridization (FISH) to detect HER2 gene overexpression in breast cancer. Breast Cancer 2015, 22:615-625.

23. Hammond ME, Hayes DF, Dowsett M, Allred DC, Hagerty KL, Badve S, Fitzgibbons PL, Francis G, Goldstein NS, Hayes M, et al: American Society of Clinical Oncology/College Of American Pathologists guideline recommendations for immunohistochemical testing of estrogen and progesterone receptors in breast cancer. J Clin Oncol 2010, 28:2784-2795.

24. Fehrenbacher L, Cecchini RS, Geyer CE, Jr., Rastogi P, Costantino JP, Atkins JN, Crown JP, Polikoff J, Boileau JF, Provencher L, et al: NSABP B-47/NRG Oncology Phase III Randomized Trial Comparing 
Adjuvant Chemotherapy With or Without Trastuzumab in High-Risk Invasive Breast Cancer Negative for HER2 by FISH and With IHC 1+ or 2. J Clin Oncol 2020, 38:444-453.

25. Charlson M, Szatrowski TP, Peterson J, Gold J: Validation of a combined comorbidity index. J Clin Epidemiol 1994, 47:1245-1251.

26. Chen JH, Yen YC, Yang HC, Liu SH, Yuan SP, Wu LL, Lee FP, Lin KC, Lai MT, Wu CC, et al: CurativeIntent Aggressive Treatment Improves Survival in Elderly Patients With Locally Advanced Head and Neck Squamous Cell Carcinoma and High Comorbidity Index. Medicine (Baltimore) 2016, 95:e3268.

27. Gralow JR, Burstein HJ, Wood W, Hortobagyi GN, Gianni L, von Minckwitz G, Buzdar AU, Smith IE, Symmans WF, Singh B, Winer EP: Preoperative therapy in invasive breast cancer: pathologic assessment and systemic therapy issues in operable disease. J Clin Oncol 2008, 26:814-819.

28. Kaufmann M, Hortobagyi GN, Goldhirsch A, Scholl S, Makris A, Valagussa P, Blohmer JU, Eiermann $\mathrm{W}$, Jackesz R, Jonat $\mathrm{W}$, et al: Recommendations from an international expert panel on the use of neoadjuvant (primary) systemic treatment of operable breast cancer: an update. J Clin Onco/2006, 24:1940-1949.

29. Shannon C, Smith I: Is there still a role for neoadjuvant therapy in breast cancer? Crit Rev Oncol Hematol 2003, 45:77-90.

30. Sparano JA, Zhao F, Martino S, Ligibel JA, Perez EA, Saphner T, Wolff AC, Sledge GW, Jr., Wood WC, Davidson NE: Long-Term Follow-Up of the E1199 Phase III Trial Evaluating the Role of Taxane and Schedule in Operable Breast Cancer. J Clin Oncol 2015, 33:2353-2360.

31. Untch M, Jackisch C, Schneeweiss A, Conrad B, Aktas B, Denkert C, Eidtmann H, Wiebringhaus H, Kummel S, Hilfrich J, et al: Nab-paclitaxel versus solvent-based paclitaxel in neoadjuvant chemotherapy for early breast cancer (GeparSepto-GBG 69): a randomised, phase 3 trial. Lancet Oncol 2016, 17:345-356.

32. Untch M, Jackisch C, Schneeweiss A, Schmatloch S, Aktas B, Denkert C, Schem C, Wiebringhaus H, Kummel S, Warm M, et al: NAB-Paclitaxel Improves Disease-Free Survival in Early Breast Cancer. GBG 69-GeparSepto. J Clin Oncol 2019, 37:2226-2234.

33. Symmans WF, Peintinger F, Hatzis C, Rajan R, Kuerer H, Valero V, Assad L, Poniecka A, Hennessy B, Green $M$, et al: Measurement of residual breast cancer burden to predict survival after neoadjuvant chemotherapy. J Clin Oncol 2007, 25:4414-4422.

34. Early Breast Cancer Trialists' Collaborative G, Darby S, McGale P, Correa C, Taylor C, Arriagada R, Clarke M, Cutter D, Davies $C$, Ewertz $M$, et al: Effect of radiotherapy after breast-conserving surgery on 10-year recurrence and 15-year breast cancer death: meta-analysis of individual patient data for 10,801 women in 17 randomised trials. Lancet 2011, 378:1707-1716.

35. Onitilo AA, Engel JM, Greenlee RT, Mukesh BN: Breast cancer subtypes based on ER/PR and Her2 expression: comparison of clinicopathologic features and survival. Clin Med Res 2009, 7:4-13.

36. Gonzalez-Angulo AM, Litton JK, Broglio KR, Meric-Bernstam F, Rakkhit R, Cardoso F, Peintinger F, Hanrahan EO, Sahin A, Guray M, et al: High risk of recurrence for patients with breast cancer who 
have human epidermal growth factor receptor 2-positive, node-negative tumors $1 \mathrm{~cm}$ or smaller. $J$ Clin Oncol 2009, 27:5700-5706.

37. Duchnowska R, Dziadziuszko R, Czartoryska-Arlukowicz B, Radecka B, Szostakiewicz B, SosinskaMielcarek K, Karpinska A, Staroslawska E, Kubiatowski T, Szczylik C: Risk factors for brain relapse in HER2-positive metastatic breast cancer patients. Breast Cancer Res Treat 2009, 117:297-303.

38. Zaheed M, Wilcken N, Willson ML, O'Connell DL, Goodwin A: Sequencing of anthracyclines and taxanes in neoadjuvant and adjuvant therapy for early breast cancer. Cochrane Database Syst Rev 2019, 2:CD012873.

39. Rusthoven CG, Rabinovitch RA, Jones BL, Koshy M, Amini A, Yeh N, Jackson MW, Fisher CM: The impact of postmastectomy and regional nodal radiation after neoadjuvant chemotherapy for clinically lymph node-positive breast cancer: a National Cancer Database (NCDB) analysis. Ann Oncol 2016, 27:818-827.

40. Hahnen E, Lederer B, Hauke J, Loibl S, Krober S, Schneeweiss A, Denkert C, Fasching PA, Blohmer JU, Jackisch C, et al: Germline Mutation Status, Pathological Complete Response, and Disease-Free Survival in Triple-Negative Breast Cancer: Secondary Analysis of the GeparSixto Randomized Clinical Trial. JAMA Oncol 2017, 3:1378-1385.

41. Loibl S, Weber KE, Timms KM, Elkin EP, Hahnen E, Fasching PA, Lederer B, Denkert C, Schneeweiss A, Braun S, et al: Survival analysis of carboplatin added to an anthracycline/taxane-based neoadjuvant chemotherapy and HRD score as predictor of response-final results from GeparSixto. Ann Oncol 2018, 29:2341-2347.

\section{Supplementary Files}

This is a list of supplementary files associated with this preprint. Click to download.

- SIFigure1.jpg

- SIFigure1.jpg

- SIFigure2.jpg

- SIFigure2.jpg 